\title{
Erratum to: Can magnifying endoscopy with narrow band imaging be useful for low grade adenomas in preoperative biopsy specimens?
}

\author{
Kazuhiro Miwa $\cdot$ Hisashi Doyama $\cdot$ Renma Ito $\cdot$ Hiroyoshi Nakanishi $\cdot$ \\ Katsura Hirano $\cdot$ Satoko Inagaki $\cdot$ Kei Tominaga $\cdot$ Naohiro Yoshida $\cdot$ \\ Kenichi Takemura Shinya Yamada Yoshibumi Kaneko $\cdot$ Kazuyoshi Katayanagi • \\ Hiroshi Kurumaya $\cdot$ Toshihide Okada $\cdot$ Masakazu Yamagishi
}

Published online: 11 February 2015

(c) The International Gastric Cancer Association and The Japanese Gastric Cancer Association 2015

\section{Erratum to: Gastric Cancer (2012) 15:170-178 \\ DOI 10.1007/s10120-011-0093-6}

In the original publication of this article, the affiliation of Kazuhiro Miwa was given as follows: Department of Gastroenterology, Ishikawa Prefectural Central Hospital,
Kanazawa, Ishikawa, Japan; and Department of Gastroenterology, Kanazawa Social Insurance Hospital, Ha-15 Okimachi, Kanazawa Ishikawa, 920-8610, Japan. The correct affiliation should include Division of Cardiovascular Medicine, Department of Internal Medicine, Kanazawa University Graduate School of Medicine.

The online version of the original article can be found under doi:10.1007/s10120-011-0093-6.

K. Miwa - H. Doyama - R. Ito - H. Nakanishi - K. Hirano ·

S. Inagaki $\cdot$ K. Tominaga $\cdot \mathrm{N}$. Yoshida $\cdot$ K. Takemura $\cdot$

S. Yamada · Y. Kaneko

Department of Gastroenterology, Ishikawa Prefectural Central

Hospital, Kanazawa, Ishikawa, Japan

K. Miwa $(\square)$

Department of Gastroenterology, Kanazawa Social Insurance

Hospital, Ha-15 Okimachi, Kanazawa, Ishikawa 920-8610,

Japan

e-mail: kzmiwatoureg@yahoo.co.jp

K. Miwa

Division of Cardiovascular Medicine, Department of Internal Medicine, Kanazawa University Graduate School of Medicine,

Kanazawa, Japan

K. Katayanagi · H. Kurumaya

Department of Pathology, Ishikawa Prefectural Central Hospital,

Kanazawa, Ishikawa, Japan

T. Okada $\cdot$ M. Yamagishi

Division of Cardiovascular Medicine, Kanazawa University,

Kanazawa, Ishikawa, Japan 